\title{
Shape-Memory Polymers for Biomedical Applications
}

\author{
Andreas Lendlein ${ }^{1,2}$ and Marc Behl ${ }^{1}$ \\ ${ }^{1}$ Institute of Polymer Research, GKSS Research Center Geesthacht GmbH \\ Kantstraße 55, 14513 Teltow, Germany, \\ ${ }^{2}$ Berlin-Brandenburg Center for Regenerative Therapies, \\ Campus Virchow Klinikum Charité, Berlin, Germany \\ to whom correspondence should be addressed: \\ andreas.lendlein@gkss.de
}

Keywords: multi-functional polymer, triple shape polymer, shape-memory polymer, biomaterial.

\begin{abstract}
Most polymers used in clinical applications today are materials that have been developed originally for application areas other than biomedicine. On the other side, different biomedical applications are demanding different combinations of material properties and functionalities. Compared to the intrinsic material properties, a functionality is not given by nature but result from the combination of the polymer architecture and a suitable process. Examples for functionalities that play a prominent role in the development of multifunctional polymers for medical applications are biofunctionality (e.g. cell or tissue specificity), degradability, or shape-memory functionality. In this sense, an important aim for developing multifunctional polymers is tailoring of biomaterials for specific biomedical applications. Here the traditional approach, which is designing a single new homo- or copolymer, reaches its limits. The strategy, that is applied here, is the development of polymer systems whose macroscopic properties can be tailored over a wide range by variation of molecular parameters.

The Shape-memory capability of a material is its ability to trigger a predefined shape change by exposure to an external stimulus. A change in shape initiated by heat is called thermally-induced shape-memory effect. Thermally, light-, and magnetically induced shape-memory polymers will be presented, that were developed especially for minimally invasive surgery and other biomedical applications. Furthermore triple-shape polymers will be introduced, that have the capability to perform two subsequent shape changes. Thus enabling more complex movements of a polymeric material.
\end{abstract}

\section{Introduction}

An actual trend in polymer science besides the development of high performance materials is the development of intelligent materials [1]. Such materials are able to build molecular machines, to act as artificial muscles or to reassemble in predefined way. It is common to most of these materials, that they display a stimuli-sensitivity towards environmental changes such as heat, light, magnetic field or the chemical environment such as $\mathrm{pH}$ or ionic strength [2].

The application of such materials are spanning from sensors and switches to intelligent fixation devices or actuators. To meet the requirements from such highly complex applications, materials are nowadays designed multifunctional. Multifunctionality is the unexpected combination of material functionalizations such as biofunctionality, degradability or shape-memory effect, e.g. dual shape capability [3]. In contrast to the intrinsic material properties a material functionality results from certain combinations of polymer architecture and specific processes. Such combinations of 
materials functionalizations are expected to open new application fields of materials [4] as the dual shape capability has been already shown to be advantageous [5]. With respect to the aging population, biomedicine is one of these application fields which are demanding new materials as most polymers used in medical applications today are materials that have been developed originally for other application areas. With the ongoing progress in biomedicine the limitations of the actually available materials are visible, challenging materials with different specifications for the properties and functionalities.

An example of a materials multifunctionality opening a new application field is the combination of biodegradability and shape-memory effect, whereby a dual-shape effect is a shape-memory effect enabling one shape change from a shape A to a shape B. This type of multifunctionality is especially advantageous for medical devices used for minimally-invasive surgery [4]. An example is a biodegradable suture material built from a dual-shape material [6]. When the material is stimulated, it is able to apply a defined stress to the wound lips which is beneficial during wound healing. The stress-level is adjusted to such a range that no necrosis of the surrounding tissue occurs and the formation of scar tissue is reduced. In the process of healing a follow-up surgery for the removal of the implant is not necessary, as the implant degrades. A strategy for the design of such tailor-made multifunctional materials is the concept of polymer systems. A polymer system consists of families of polymers, in which the macroscopic properties, such as thermal or mechanical properties, can be controlled almost independently from each other over a wide range, by the systematic variation of specific molecular parameters. Consequently, multifunctional materials requiring a specific combination for a certain application can be obtained by only a slight variation of the molecular structure or chemical composition in such a polymer system [7-9].

While biodegradability of multifunctional materials is achieved by the incorporation of hydrolysable bonds as weak links, which are cleaved by water under the physiological conditions $[6,10]$, the material's movement is realized by the dual-shape capability. Dual-shape materials transform from a shape A into a shape B exposed to a suitable stimulus $[11,12]$. Shape $A$ is a temporary shape created by a programming process, and shape $\mathrm{B}$ is a permanent shape obtained during the initial polymer processing. The programming process consists of a mechanical deformation and subsequent fixation of this deformation. When exposed to an external stimulus, shape-memory polymers recover their permanent shape. This process of programming and recovery can be repeated several times with different temporary shapes in subsequent cycles.

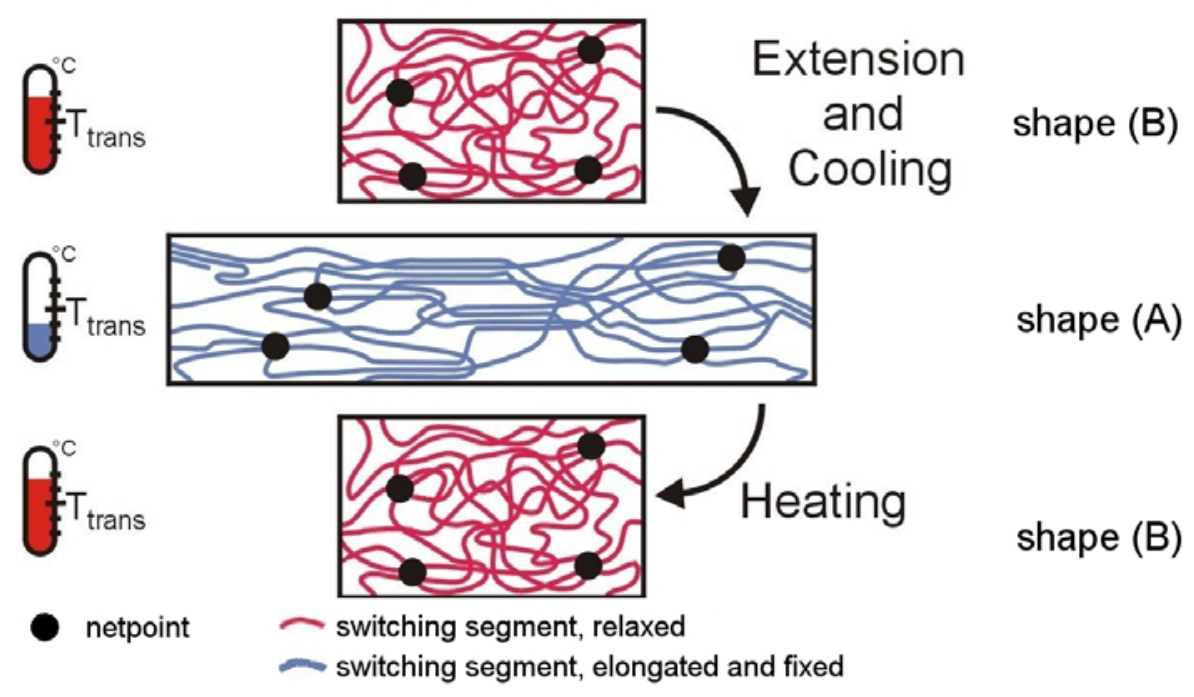

Fig. 1: Molecular mechanism of the thermally-induced shape-memory effect (here dual-shape effect)

$\mathrm{T}_{\text {trans }}=$ thermal transition temperature related to the switching phase (taken from [11];Copyright 2002 Wiley-VCH, Germany) 


\section{Dual-Shape Polymers}

Dual-shape polymers which are shape-memory polymers enabling one shape change, are elastic polymer networks being equipped with suitable stimuli-sensitive switches. In Fig. 1 a scheme for such polymer networks consisting of molecular switches (lines in Fig. 1) and netpoints (dots in Fig. 1 ) is depicted. The permanent shape of the polymer network is determined by chemical netpoints or physical interactions. Physically crosslinked dual shape polymers require a morphology which consists of at least two segregated domains, which can be provided for example by block copolymers. While the molecular switches are consisting of domains formed by chain segments related to the second highest thermal transition ( $\left.T_{\text {trans }}\right)$ (switching segment), the netpoints are formed by domains with the highest thermal transition (hard segment).

The dual shape effect is quantified in cyclic, thermomechanical tests by means of a tensile tester under strain- or stress-control [11, 13]. While in the strain-controlled tests the stress on the specimen is recorded, in stress-controlled tests vice versa the strain of the specimen is recorded. A single cycle includes programming of the test piece and recovering of its permanent shape. The strain-controlled test consists of four steps: (1) heating of the sample to the upper working temperature $T_{\text {high }}$ which at the same time is the recovery temperature, deformation of the sample to a certain extension $\left(\varepsilon_{\mathrm{m}}\right)$ at a defined deformation rate; (2) cooling to the lower working temperature $\mathrm{T}_{\text {low }}$ while $\varepsilon_{\mathrm{m}}$ is kept constant; (3) unloading of the specimen to $0 \%$ extension at $\mathrm{T}_{\text {low }}$; (4) go back to (1). In these tests the strain applied to the sample with respect to the relative distance of the clamps of the tensile tester is controlled while the evolving stress is recorded. $T_{\text {high }}$ and $T_{\text {low }}$ are held constant at least for 10 min before loading or unloading of the specimen. $\mathrm{T}_{\text {high }}$ and $\mathrm{T}_{\text {low }}$ are typically adjusted to $\mathrm{T}_{\text {trans }} \pm(20-30) \mathrm{K}$ of the investigated polymer network. The cyclic thermomechanical tests are usually performed three to five times. While the first cycle erases irregularities arising from the polymers thermal history, the other cycles are used for the quantification. Besides stress- or strain-controlled protocols different procedures of sample programming are possible (cold drawing at $\mathrm{T}<\mathrm{T}_{\text {trans }}$ or temporarily heating up of the test piece to $\mathrm{T}>\mathrm{T}$ trans).

To broaden the application spectrum of multifunctional materials, recent research is focussing on the implementation of other stimuli than heat. This was achieved by indirect actuation of the dualshape effect or the implementation of molecular switches, which are sensitive to other stimuli other than heat. Incorporation of magnetic nanoparticles of iron(III)oxide cores in a silica matrix into shape-memory thermoplasts enables remote actuation of the thermally-induced dual-shape effect in alternating magnetic fields (Fig 2) [14]. While this effect still relies on the thermal actuation of the dual shape effect, incorporation of reversibly reacting molecular switches enables light-induced stimulation of dual-shape polymers [15-17] which is independent of any temperature effects. Cinnamic acid (CA) or cinnamyliden acetic acid (CAA) [15] moieties have been used as the lightsensitive molecular switches. Upon irradiation with suitable wavelengths these functional groups form covalent bonds with each other, which can be cleaved again, when the newly formed bonds are irradiated with other suitable wavelengths. 


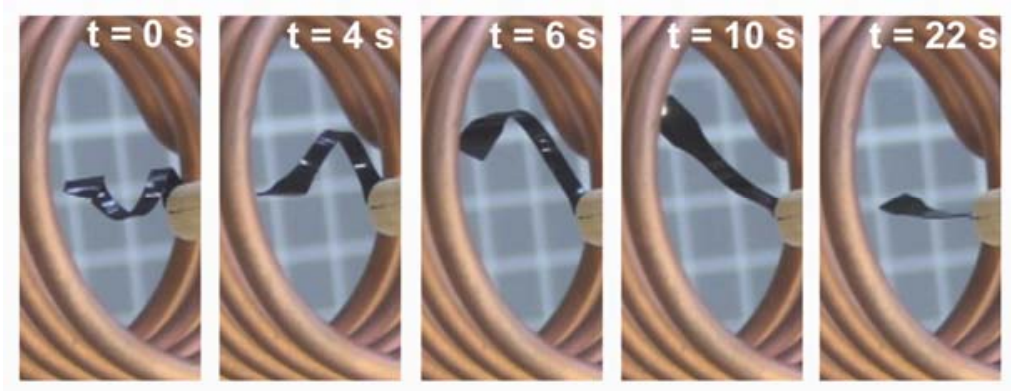

Fig. 2: Magnetically-induced shape-memory effect of a thermoplastic shape-memory composite from nanoparticles consisting of iron(III)oxide particles in a silica matrix and a polyetherurethane. (taken from [14]; Copyright 2006 National Academy of Sciences, U.S.A)

\section{Triple Shape Materials}

In triple shape materials two subsequent shape changes have been implemented in polymeric material $[18,19]$. Besides three individual shapes such a material allows by individual choice of the transition temperatures two dual shape effects. On the molecular level this has been achieved by the integration of a second switching segment into the polymer network which provides a least two segregated domains resulting in two transition temperatures $T_{\text {trans, } A}$ and $T_{\text {trans,B. While the netpoints }}$ resulting from the crosslinking reaction define the original shape $(C)$, shapes $(A)$ and $(B)$ are created in a two-step thermomechanical programming process. The physical crosslinks are associated to the highest transition temperature $T_{\text {trans,B }}$ and provide shape (B), while shape (A) relates to the second highest transition temperature $T_{\text {trans,A }}$. In the course of programming such a polymer network is heated to $T_{\text {high }}$ where the material is in the elastic state and is deformed. The cooling under external stress to $T_{\text {mid }}\left(T_{\text {trans,A }}<T_{\text {mid }}<T_{\text {trans,B }}\right)$ results in formation of physical crosslinks related to $T_{\text {trans, } B}$. When the external stress is released, shape B is obtained. Subsequent deformation of this shape at $\mathrm{T}_{\text {mid }}$ and cooling to $\mathrm{T}_{\text {low }}$ under external stress creates shape $\mathrm{A}$. Reheating to $\mathrm{T}_{\text {mid }}$ recovers shape $\mathrm{B}$ and subsequent heating to $\mathrm{T}_{\text {high }}$ results in shape $\mathrm{C}$. The triple shape effect is shown for an intelligent tube as demonstration object in Fig. 3. 


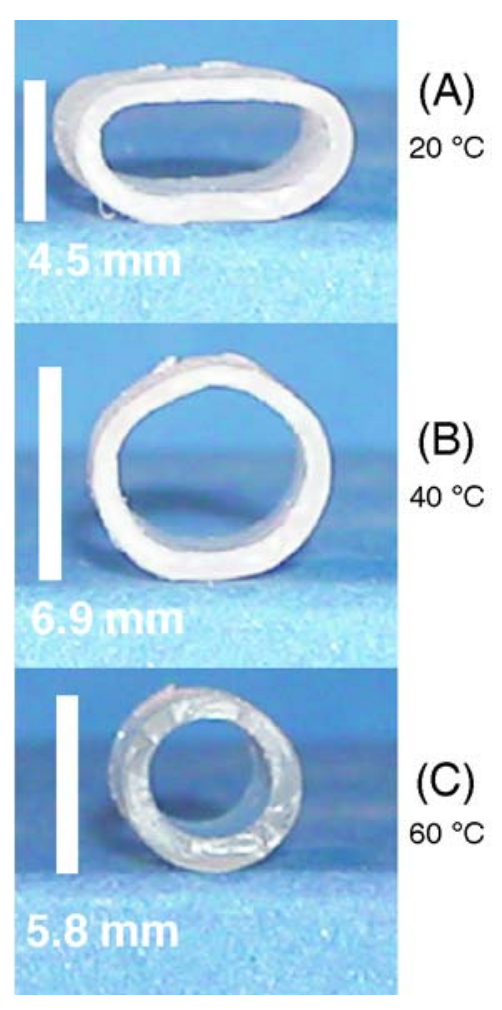

Fig. 3: Series of photographs illustrating the triple shape effect of an intelligent tube as demonstration object prepared from CLEG(50): The picture series show the recovery of shapes (B) and (C) by subsequent heating to 40 and $60^{\circ} \mathrm{C}$, beginning from shape $(\mathrm{A})$, which was obtained as a result of the two-step programming process.

(taken from [18], Copyright 2006 National Academy of Sciences, U.S.A)

The generality of this approach has been shown by two independent polymer networks architectures, which were developed (Fig. 4) by photo-induced copolymerization of a methacrylate-

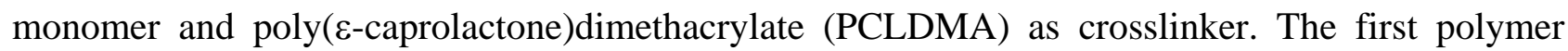
network system, named MACL, contains poly( $\varepsilon$-caprolactone)-segments (PCL-segments) and poly(cyclohexyl methacrylate)-segments (PCHMA-segments). Both types of polymer chain segments form links between netpoints and contribute in this way to the overall elasticity of the polymer network. In the second polymer network system, called CLEG, poly(ethylene glycol)segments (PEG-segments) are introduced as side chains having one dangling end, while PCLsegments connect two netpoints and mainly determine the elasticity of the polymer network. In $C L E G$-networks $T_{\text {trans,B }}$ and $T_{\text {trans,A }}$ are melting temperatures, in MACL $T_{\text {trans,A }}$ is a melting and $T_{\text {trans,B }}$ a glass transition temperature.

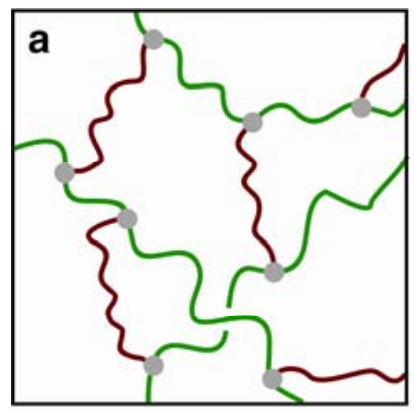

MACL

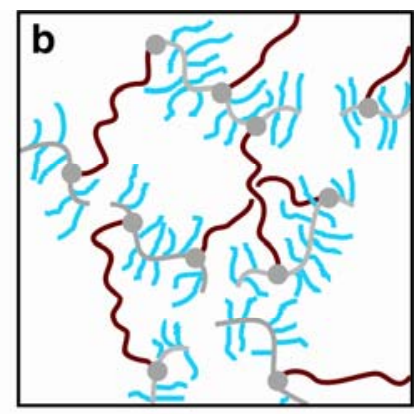

CLEG

Fig. 4: Polymer network architecture. a, MACL-network. b, CLEG-network. Green - PCHMA segments; red - PCL segments; blue - PEG side chains; grey - crosslinks. (taken from [18]; Copyright 2006 National Academy of Sciences, U.S.A) 
As mentioned above, the triple shape properties of all investigated materials can also be used as dual shape materials in which $T_{\text {trans }}$ can be varied according to the specific requirements as the dual shape experiments can be performed either between $T_{\text {low }}$ and $T_{\text {mid }}$ using $T_{\text {trans,A }}$ or between $T_{\text {mid }}$ and $T_{\text {high }}$ using $T_{\text {trans,B }}$ [19]. Additionally, a combination of both switching phases is possible. The temperature range between $40{ }^{\circ} \mathrm{C}$ and $70{ }^{\circ} \mathrm{C}$ (case I) allows determination of the capability of the PCL phase to act as switching phase. The ability of the PEG phase as switching phase can be determined between $0{ }^{\circ} \mathrm{C}$ and $40^{\circ} \mathrm{C}$ (case II). Both crystallizable phases can support the fixation of a temporary shape if the cyclic, thermomechanical experiments are conducted between $0{ }^{\circ} \mathrm{C}$ and 70 ${ }^{\circ} \mathrm{C}$ (case III). While in case $\mathrm{I}_{\mathrm{sw}}$ is in the range of $\mathrm{T}_{\mathrm{m}}(\mathrm{PCL})$, values for $\mathrm{T}_{\mathrm{sw}}$ are similar to the values determined for $T_{\mathrm{m}}$ (PEG) in case II. In case III, the crystallization of PCL already fixes the deformation which led to the orientation of PCL chain segments. Therefore, $\mathrm{T}_{\mathrm{sw}}$ is in the range of $T_{\mathrm{m}}(\mathrm{PCL})$ and no additional step in the recovery curve indicating a recovery triggered by melting of PEG crystallites can be detected.

\section{Summary}

The shape-memory effect is presented as an example for a material functionality, which can be implemented in multifunctional materials. With the realization of other stimuli different from heat, this technology platform is substantially broadened. In addition, a triple shape effect is introduced which enables two subsequent shape changes occurring at two distinct switching temperatures. Triple shape materials can also be programmed as dual shape materials offering the choice of the transition temperature by the proper selection of programming parameters. Potential applications for this technology are especially in the area of medicine, e.g. minimally-invasive surgery.

\section{References}

[1] M. Behl, R. Langer, A. Lendlein in M. Shahinpoor: H.-J. Schneider: Intelligent Materials. (Royal Society of Chemistry, UK, 2008)

[2] M. Behl, A. Lendlein: Soft Matter, Vol. 3 (2007), p. 58.

[3] A. Lendlein, S. Kelch: Functionally Graded Materials Viii, Vol. 492-493 (2005), p. 219.

[4] F. El Feninat, G. Laroche, M. Fiset, D. Mantovan: Adv. Eng. Mater., Vol. 4 (2002), p. 91.

[5] F. Metzger, T. S. Wilson, D. Schumann, D. L. Matthews, D.J. Maitland: Laser. Surg. Med., Vol. 30 (2002), p. 1.

[6] A. Lendlein, R. Langer: Science, Vol. 296 (2002), p. 1673. 
[7] A. Lendlein, A.M. Schmidt, M. Schroeter, R. Langer: J. Polym. Sci. Pol. Chem., Vol. 43 (2005), p. 1369.

[8] A. Alteheld, Y. Feng, S. Kelch, A. Lendlein: Angew. Chem. Int. Edit., Vol. 44 (2005), p. 1188.

[9] N.Y. Choi, S. Kelch, A. Lendlein: Adv. Eng. Mater., Vol. 8 (2006), p. 439.

[10] A. Lendlein, A.M. Schmidt, R. Langer: P. Natl. Acad. Sci. USA, Vol. 98 (2001), p. 842.

[11] A. Lendlein, S. Kelch: Angew. Chem. Int. Edit., Vol. 41 (2002), p. 2034.

[12] M. Behl, A. Lendlein: Materials Today, Vol. 10 (2007), p. 20.

[13] M. Behl, A. Lendlein: Shape-memory Polymers, in McGraw-Hill: Yearbook of Science \& Technology, (S.S.E. Jonathan Weil, United States of America, 2007).

[14] R. Mohr, K. Kratz, T. Weigel, M. Lucka-Gabor, M. Moneke, A. Lendlein: P. Natl. Acad. Sci. USA, Vol. 103 (2006), p. 3540.

[15] A. Lendlein, H. Jiang, O. Jünger, R. Langer: Nature, Vol. 434 (2005), p. 879.

[16] H.Y. Jiang, S. Kelch, A. Lendlein, Adv. Mater., Vol. 18 (2006), p. 1471

[17] Y.L. Yu, T. Ikeda, Macromol. Chem. Physic., Vol. 206 (2005), p. 1705.

[18] I. Bellin, S. Kelch, R. Langer, A. Lendlein , P. Natl. Acad. Sci. USA, Vol. 103 (2006), p. 18043.

[19] I. Bellin, S. Kelch, A. Lendlein, J. Mater. Chem., Vol. 17 (2007), p. 2885 
